\title{
À la frontière des autres genres : les Élégies de l'abbé Le Blanc
}

\author{
Nicholas Dion \\ Université de Sherbrooke
}

Les nombreuses querelles littéraires qui ont scandé les XVIIe et XVIIIe siècles en France ont souvent illustré, chacune à leur manière, dans quelle mesure les frontières entre les différents genres s'avèrent poreuses: de la tragédie régulière à l'opéra français en passant par le roman héroïque et la nouvelle historique, l'entreprise définitoire de l'âge classique s'est interrogée à maintes reprises sur les critères génériques qui servent à délimiter les œuvres. L'élégie n'a pas échappé à cette réflexion. De fait, la première adaptation qu'a subie le genre lors 
de son passage du latin au français en a fortement ébranlé les limites : puisque le seul critère servant à définir l'élégie antique, le distique élégiaque, était tributaire de la prosodie latine, l'élégie française se retrouvait sans champ propre, sans frontières spécifiques. Ainsi, lorsque les premiers théoriciens du genre élégiaque français du XVIIIe siècle ont abordé la question, en particulier les abbés Le Blanc (1731) et Michault (1734), ils ont multiplié les comparaisons entre l'élégie et les autres genres afin d'en établir les nuances, les spécificités et les bornes. Le projet de Le Blanc s'avère particulièrement significatif dans la mesure où non seulement l'auteur publie le premier traité consacré exclusivement à l'élégie française en près d'un siècle, mais encore il l'accompagne d'une douzaine d'élégies de sa plume, censées exemplifier ses positions théoriques. De la lecture de son "Discours sur l'élégie» se dégage cependant une rhétorique qui gomme paradoxalement les distinctions génériques alors même qu'elle devait assurer leur valeur discriminante. Ces nombreuses imprécisions informent d'autant plus la composition des douze élégies que ces dernières se veulent une tentative de repousser les limites imposées au genre élégiaque dans la tradition française telle que perçue et analysée par l'abbé Le Blanc.

\section{Entre la joie et la tristesse}

L'élégie française n'est certes pas une invention du XVIIIe siècle. À l'instar de plusieurs autres genres, elle prend ses racines à la Renaissance, dans la foulée de la revalorisation des œuvres et des genres antiques. Or l'élégie gréco-latine désigne avant tout un mètre, le distique élégiaque, et bien que la triade Tibulle, Properce et Ovide donne l'impression d'une certaine 
homogénéité générique - encore que l'on doive nuancer un tel propos $^{1}-$, les poètes grecs et latins ont en réalité composé des poèmes élégiaques sur presque tous les sujets : la guerre, les préceptes moraux, le calendrier des fêtes religieuses, les mathématiques, etc ${ }^{2}$. Puisque la versification française s'appuie sur le nombre des syllabes et non sur leur quantité, elle rendait l'importation du distique élégiaque difficile, voire impossible: tout au plus note-t-on, au fil des siècles, quelques tentatives infructueuses et sans véritable suite de reproduire le rythme inégal engendré par l'alternance d'un hexamètre et d'un pentamètre dactyliques, comme c'est le cas, par exemple, chez Pierre de Ronsard, Joachim du Bellay et Rémy Belleau. De fait, la forme d'une suite de vers d'égale longueur (l'octosyllabe, mais surtout le décasyllabe et l'alexandrin) s'impose rapidement dans la pratique. L'élégie française devait désormais trouver sa spécificité dans ses sujets, dans sa matière, et non dans sa forme, beaucoup trop commune. Autrement dit, les critères servant à la délimiter s'inversent complètement : d'un genre à la versification précise et à la thématique étendue, l'élégie devient ainsi un poème sans forme particulière ou même fixe, mais possédant une matière plus restreinte.

Une révolution générique de cette envergure ne se réalise pas sans heurts. Cependant, un consensus à propos des traits caractéristiques de l'élégie française - ou du caractère élégiaque, pour reprendre le titre du premier traité consacré à

${ }^{1}$ Les traités néolatins des siècles classiques ne font pas consensus sur la place que doivent tenir Catulle ou certains recueils ovidiens (Les héroïdes, Les fastes, et parfois même Les tristes). Sur ce point, les théoriciens français des XVIIe et XVIII e siècles sont leurs directs héritiers.

2 À ce propos, voir Laure Chappuis Sandoz (2011), en particulier la savante introduction : «Au-delà de l'élégie d'amour : limites et ambitions du genre », p. 11-26. 
l'élégie française, celui que publie La Mesnardière en 1640 - se cristallise très rapidement autour de l'idée de tristesse (voir Dion, 2013, p. 65-84). Sans trop nous y attarder, rappelons que si les traités de poétique de la Renaissance glosent chacun à sa manière la définition donnée par Horace dans son Art poétique, force est de constater qu'ils infléchissent considérablement le texte latin afin de légitimer l'existence d'une élégie qui ne soit pas composée versibus impariter. Les différents traités de Sébillet, de Pierre de Laudun d'Aigaliers et de Vauquelin de La Fresnaye, par exemple, posent au demeurant les germes d'une réflexion qui opère par comparaison et qui peine à tracer les frontières de l'élégie : tour à tour, elle se voit comparée à ou confondue avec d'autres genres comme l'épître, le dialogue, la farce, la complainte, voire le lai ${ }^{3}$. Le traité de La Mesnardière, quant à lui, peine à articuler l'héritage gréco-latin aux pratiques françaises. L'exemple du «Calianthe victorieux, mais blessé à mort pour la querelle de sa Maîtresse, \& mourant d'amour \& de douleur ", poème que l'auteur place à la fin de son traité en tant que modèle à suivre, est fort révélateur, car La Mesnardière s'éloigne à la fois du fond des œuvres antiques - le cadre narratif annoncé dans le titre possède peu d'équivalents antiques - et de leur forme : un personnage fictif y chante ses propres douleurs alors que l'élégie appartient traditionnellement à la poésie personnelle 4 . Mais dans l'ensemble, les théoriciens s'entendent sur un point: le ton qu'emprunte l'élégie doit être triste.

\footnotetext{
3 Respectivement dans Thomas Sébillet (1990), p. 128-129; Pierre de Laudun d'Aigaliers (2000), p.68, 69 et 77; Jean Vauquelin de La Fresnaye (1885), p. 29 , v. 523 sq.

4 C'est d'ailleurs souvent à ce titre que certains théoriciens de l'âge classique refusent le titre d'élégies aux Héroïdes d'Ovide; nous y reviendrons.
} 
À la fin du XVIIe siècle, une telle conception pose de plus en plus de problèmes, entre autres parce que les critiques doivent composer avec le célèbre vers d'Horace que Boileau traduit ainsi : "Elle [l'élégie] peint des amants la joie et la tristesse. » (1998, p. 94, chant II, v. 41) Or, dans un contexte où la métrique ne possède pas de valeur discriminante, peindre la joie et la tristesse reviendrait à élargir le champ de l'élégie jusqu'à son point de rupture, à faire éclater ses limites. Un seul exemple, particulièrement éloquent pour notre propos, suffit à illustrer la confusion qui règne autour du genre élégiaque : la «Description de l'empire de la poésie » de Fontenelle, publiée à l'origine dans le Mercure galant de janvier 1678. Dans cet exercice de topographie allégorique, l'élégie est située sans grande surprise dans les Provinces de basse poésie, plus particulièrement aux confins des Déserts du bon sens, dans la Province des pensées fausses. La description de la région et la position privilégiée du genre élégiaque, quant à elles, étonnent davantage :

On n'y marche que sur les fleurs; tout y rit, tout y paroît enchanté : mais ce qu'il y a d'incommode, c'est que la terre n'en étant pas solide, on y enfonce partout, \& on n'y sauroit tenir pied. L'Élégie en est la principale Ville : on n'y entend que des gens plaintifs; mais on diroit qu'ils se jouent en se plaignant. La Ville est toute environnée de bois \& de rochers, où les habitans vont se promener seuls; ils les prennent pour confidens de tous leurs secrets; \& ils ont tant de peur d'être trahis, qu'ils leur recommandent souvent le silence ${ }^{5}$. (Bernard Le Bovier de Fontenelle, 1766, p. 438)

Passons sur la critique des topoï de l'élégie, maintes fois ridiculisés par les théoriciens, qui voient dans les apostrophes

\footnotetext{
5 Pour la carte, voir « Description de l'empire de la poésie » (1969), p. 22-24; la partie du texte portant sur l'élégie a été élaguée.
} 
aux objets inanimés une entorse à la vraisemblance classique, d'autant plus qu'elles impliquent un autre brouillage générique, cette fois avec l'églogue. En revanche, poser le genre élégiaque comme chef-lieu d'une contrée où tout rit, où tout paraît enchanté, atteste sans contredit la perméabilité des frontières génériques et des catégories esthétiques. Fontenelle introduit de surcroît une précision considérable: les habitants sont plaintifs, "mais on diroit qu'ils se jouent en se plaignant». La remarque évoque à la fois l'affectation et l'emphase que l'on reproche parfois au genre, notamment aux mauvaises élégies, et la délectation mélancolique associée aux douces peines amoureuses. "La douleur qu'on veut calmer, on la charge d'abord, on s'en occupe, on s'en remplit, on s'y plaist », résume l'abbé Fraguier (1729, p. 280). Une certaine joie se mêle donc effectivement à la tristesse, en parfait accord avec l'exégèse horatienne proposée par Boileau'.

\section{Tracer les insaisissables frontières de l'élégie française}

Les frontières de l'élégie française ont toujours été poreuses, ce qui s'explique aisément par la spécificité de la prosodie française, mais également par l'absence d'assises théoriques claires relevant d'une autorité soit critique, soit littéraire: l'élégie aux siècles classiques ne possède pas de parangon. À l'instar des appréciations contradictoires formulées à l'égard des élégiaques latins, aucun poète français ne peut prétendre servir de modèle en matière d'élégie si l'on en croit le jugement des différents commentateurs. Qui plus est, après la publication du traité de La Mesnardière, aucun ouvrage ne se penche sur le

${ }^{6}$ Et entérinée par André Dacier. Voir Dion (2013). 
sujet en près d'un siècle, divers commentaires assez laconiques étant plutôt disséminés dans des recueils, dans des anthologies ou dans des dictionnaires. En effet, il faut attendre les Élégies de l'abbé Jean-Bernard Le Blanc en 1731 pour que soit de nouveau abordée la poétique de l'élégie française et non plus uniquement celle de l'élégie antique.

Tenant lieu de préface, le «Discours sur l'élégie » que l'auteur place en tête du volume de ses œuvres - le recueil compte également quelques odes, sonnets et poésies de circonstances - s'inspire certes de quelques traités savants ${ }^{7}$, mais il tente avant tout de remettre en question la doxa qui entoure la conception du genre et d'instituer une nouvelle forme d'élégie. Ce sont donc à la fois la partie théorique et l'exercice poétique qui pallient un manque: d'une part, «la véritable nature de l'Élégie n'est pas assez connüe » et, d'autre part, «malgré le grand nombre qu'on en a fait, on en trouve peu que l'on puisse lire sans ennui» (Le Blanc, 1731, p. 2-3). Cependant, à trop vouloir faire œuvre originale et forcer les comparaisons hardies, l'auteur repousse les frontières du genre élégiaque au point qu'elles deviennent accessoires, pour ne pas dire incohérentes. Si tout poème est susceptible d'être considéré comme une élégie, et si un poème élégiaque peut emprunter la forme, le sujet et le ton qui lui plaît, à quoi sert la typologie poétique?

À première vue, sa définition paraît pourtant conventionnelle : l'élégie «n'est autre chose que la plainte d'un

7 Par exemple, dès les premières pages, il plagie mot pour mot l'abbé Fraguier : «la plainte si naturelle à l'homme est un grand fond pour la Poësie; \& qu'est-ce qu'une Elégie? c'est un Poëme triste \& plaintif » (Le Blanc, 1731, p. 3). Voir Fraguier (1729), p. 277. 
amour mécontent » (Le Blanc, 1731, p. 7). En réalité, on sent que Le Blanc hésite entre deux voies ${ }^{8}$ : l'héroïde, d'après le modèle ovidien, et la tirade tragique, fort probablement inspirée de l'esthétique racinienne et de son interprétation'. Aussi tente-t-il d'emblée d'étendre les bornes du domaine élégiaque :

Il y a des gens qui au sujet de l'Élégie donnent dans une erreur à mon avis bien singuliére; ils s'imaginent qu'une Épitre amoureuse, quoique triste, n'est pas une Élégie, sur tout si elle est héroïque : Comme si l'Élégiaque n'étoit pas aussi susceptible de l'héroïque que du familier. (Le Blanc, 1731, p. 4)

Que l'épître amoureuse puisse être une élégie, voilà un point sur lequel s'entendent presque tous les traités néolatins et français sur une période d'environ deux cents ans. En revanche, le genre élégiaque appartient depuis toujours au style simple, que Le Blanc assimile en l'occurrence au "familier »; c'est d'ailleurs l'une des constantes stylistiques que l'on trouve chez les commentateurs, car quoique le genre élégiaque pose problème, son style fait habituellement consensus. L'héroïque, qui caractérise l'épopée ou la tragédie, relève en contrepartie du style élevé et ne saurait que difficilement qualifier l'élégie. C'est moins le théâtre tragique que les Héroïdes d'Ovide qui sont ciblées ici, comme le confirme un passage ultérieur où Le Blanc se livre à un rapide survol de la production élégiaque antique. Après avoir refusé à Catulle le titre d'élégiaque puis remis en

8 Il s'agit des deux cas les plus significatifs et l'on aurait beau jeu de multiplier les exemples, comme ce passage à propos de la pastorale: "Le sujet des Élégies peut être plus varié qu'on ne pense; car bien que toutes les Églogues ne soient pas des Élégies, il y a bien des Élégies qui peuvent être en même temps des Églogues : Il suffit pour cela d'y faire parler des Bergers amoureux d'un ton plaintif. » (Le Blanc, 1731, p. 19) Qui trop embrasse mal étreint, en critique comme ailleurs.

${ }_{9}$ À ce sujet, voir Dion (2012), ch. 3 et ch. 9. 
question les caractères de Tibulle et de Properce, il conclut «qu'Ovide doit occuper le premier rang» (Le Blanc, 1731, p. 22). Il précise néanmoins :

Les véritables Élégies d'Ovide sont celles qui n'en portent pas le nom \& qu'il a intitulées Héroïdes. C'est-là qu'il a peint l'amour tel qu'il doit être pour faire la matiere de la belle Élégie: l'infortunée Didon, la fidelle Pénéloppe, la furieuse Médée s'y plaignent de leurs malheurs, s'y plaignent de leur amour. (1731, p. 22)

Ce faisant, il s'inscrit en faux contre l'ensemble de la tradition antique et de ses commentateurs modernes: les épîtres héroïdes d'Ovide sont souvent incluses dans le corpus élégiaque latin - elles sont bel et bien composées en distiques élégiaques -, mais elles n'en constituent en aucun cas le canon ${ }^{10}$. Le Blanc va d'ailleurs plus loin :

Mais de même que les Stances ne conviennent pas à la véritable Élégie, il me semble aussi que les Anciens ont eû tort de choisir les Vers Élégiaques, comme les plus propres à peindre les passions amoureuses; ils sont trop affectés, \& trop badins, je ne les trouve bons que pour les Élégies de l'Art d'aimer, dictées plûtôt par l'esprit que par le cœur; mais pour les autres je crois que le Vers Héroïque ou l'Iambe étoient les seuls qui leur pussent convenir. (Le Blanc, 1731, p. 23)

Singulière relecture de l'histoire qui, sous couvert de corriger les Anciens, s'enferre dans une contradiction aux limites de l'hystérologie : si l'élégie est apparue, c'est d'abord parce qu'il y a eu un vers élégiaque. Selon la logique de Le Blanc, même les seules «véritables élégies » du poète élégiaque occupant «le premier rang » ne posséderaient pas une forme propre au sujet

10 Soulignons au demeurant que les adjectifs antéposés témoignent de l'infléchissement général que Le Blanc souhaite apporter au genre : bien que conventionnelle et relevant des manuels de rhétorique, la formulation « furieuse Médée » ne s'applique guère à l’héroïde en question. 
élégiaque par excellence, la peinture des passions amoureuses. De surcroît, étant donné que les élégies françaises sont majoritairement composées en alexandrins - le vers héroïque français et le substitut de l'iambe, pied de base du dialogue des pièces de théâtre gréco-latine-, cela revient à affirmer sa supériorité.

Il en va de même pour les parallèles que l'auteur dresse entre l'élégie et la tragédie. Dans un premier temps, Le Blanc entérine le célèbre jugement formulé par l'abbé Villars à propos de Bérénice de Jean Racine :

Qu'on sépare de celles de Racine quelques Monologues qui ne roulent que sur l'amour, ce seront autant d'Élégies; cependant quel intérêt n'y prend-t-on pas? Jusqu'à quel point ne s'en laisse-t-on pas toucher? Bérénice n'est autre chose qu'une longue \& magnifique Élégie que ce grand Poëte a mise sur le Théatre. (Le Blanc, 1731, p. 7-8)

Il passe d'ailleurs rapidement du particulier au général : ce qui avait été dit d'une pièce vaut maintenant pour tout le corpus racinien. Après avoir affirmé que l'élégie « est une sorte de diminutif» de la tragédie, il avance même qu'elle relève d'impératifs esthétiques semblables : "elle ne peut être intêressante qu'autant qu'elle est la representation d'une action. » (Le Blanc, 1731, p. 13) Elle constitue donc une imitation au sens aristotélicien du terme, ce que confirme la suite du passage décrivant la forme idéale qu'elle doit revêtir: «elle n'en doit pas moins avoir son exposition, son accroissement \& sa fin. » (Le Blanc, 1731, p. 14) La conclusion de son raisonnement s'avère pour le moins surprenante :

Car dès qu'il y aura de l'amour, vous pouvez faire entrer dans l'Élégie les plus grands traits de l'Histoire, bien plus aisément que dans la Tragédie où l'on est obligé d'imaginer des circonstances qui multiplient les Scènes \& prolongent l'action. (Le Blanc, 1731, p. 18) 
Aussi l'élégie est-elle envisagée non seulement comme une variante abrégée de la tragédie, mais encore comme une version condensée pouvant faire l'économie des parties moins intéressantes, au sens classique du terme. Le poème élégiaque, s'appuyant sur la narration, est susceptible d'exprimer la plainte amoureuse et d'en présenter la genèse dans un même mouvement afin de mieux toucher le lecteur. C'est du moins ce que laisse entendre le dialogue que Le Blanc engage quelques pages plus loin avec l'abbé Fraguier à propos d'une éventuelle catharsis élégiaque (Le Blanc, 1731, p. 14 et suivantes).

\section{Fureurs élégiaques}

Pourquoi tant insister sur les liens qui unissent l'élégie française aux autres genres poétiques? Pour quelle raison infléchir à ce point la lecture de son origine, de son évolution et du domaine qu'elle doit occuper au Royaume de la poésie? À vrai dire, Le Blanc s'intéresse moins aux catégories esthétiques elles-mêmes et à leurs limites qu'à la matière qu'il souhaite exploiter dans ses poèmes : la fureur amoureuse. Rappelons-le : le «Discours» de l'abbé Le Blanc est le premier texte d'importance consacré à l'élégie française au XVIIIe siècle, le deuxième seulement en près d'un siècle et le second depuis l'apparition du genre à la Renaissance. Pourtant, il serait de moindre importance s'il n'était suivi des douze élégies composées par le même auteur. Ainsi, son approche est directement informée par une pratique qui entend évacuer la tendresse, l'attribut premier du style de l'élégie au XVIIe siècle (voir Denis, 2008, p. 74 sq), non pour des raisons théoriques, mais pour des impératifs pratiques : 
Ainsi vous êtes maître de choisir; une passion tendre pourra plaire; mais une vive peinture des violences de l'amour, les excès, les emportements, la fureur même toucheront, frapperont davantage. C'est par ses images effrayantes qu'on répand dans l'esprit du Lecteur des idées qui le réveillent, qui l'attachent \& qui par là ne peuvent manquer de lui plaire. (Le Blanc, 1731, p. 42-43)

Et l'auteur de prendre pour exemple une série de personnages tragiques, tous raciniens à une exception près : Didon, Oreste, Ariane, Junie, Hermione. Et si les «images effrayantes» détonnent dans un traité portant sur l'élégie, le modèle que choisit l'auteur est tout aussi singulier et entraîne un énième parallèle avec la tragédie : «j'ose assurer qu'aucun n'a réüssi à peindre la violence des Passions comme M. de Crébillon, il ne s'est pas contenté d'exciter la pitié, il a cherché à répandre la terreur dans les esprits. » (Le Blanc, 1731, p.47) Un poète élégiaque qui se réclame de Crébillon père et de son théâtre, voilà qui promet de singulières élégies. De fait, les compositions de l'abbé Le Blanc repoussent les frontières du genre élégiaque et défient presque toute catégorisation tellement elles forment un ensemble hétérogène. Prenons par exemple l'incipit du premier poème :

Amour! unique Autheur des tourmens que j'endure,

Si tu me fais verser des pleurs pour un Parjure,

Cruel Amour! du moins laisse-moi lui cacher

Des maux qui désormais ne le peuvent toucher. (Le Blanc, 1731, p. 67)

L'apostrophe à la passion amoureuse et sa personnification sont des lieux communs, tout comme le souhait du personnage de dérober ses larmes; elles respectent bel et bien la poétique élégiaque. En revanche, l'infléchissement des topoï pastoraux que l'on trouve plus loin se révèle fort original : 
Ma fureur malgré moi surmonte ma foiblesse, Sans savoir où je suis, sans savoir où je vais, Je me jette au travers des Bois les plus épais. Je cours de tous côtez. Une ronce, un branchage Ensanglante mon bras, déchire mon visage; Mais livrée aux tourmens que mon cœur souffre alors, Je ne vois, ne connois, ne sens que mes transports. Le doux chant des Oiseaux qui dans ces Lieux habitent, Ne fait qu'acroître encor les troubles qui m'agitent; Leurs plaisirs, leurs amours irritent ma fureur; Et je les interromps par des cris pleins d'horreur. (Le Blanc, 1731, p. 69-70)

Encadrés par des mentions de la fureur du personnage, les lieux communs sylvestres n'apparaissent que pour mieux être retournés. Les bois, loin de calmer les tourments passionnels, les exacerbent physiquement («ensanglante», «déchire») et psychologiquement («acroître », «irrite»), alors qu'Ériphile terrorise de pauvres volatiles. Crébillon n'aurait certes pas désavoué ces vers ${ }^{11}$.

À la limite, un tel poème pourrait se lire comme une tentative de jouer avec l'horizon d'attente du lecteur et d'ainsi créer un effet de surprise en exploitant les loci élégiaques de manière originale. Mais qu'en est-il des motifs étrangers, presque contraires à la poétique élégiaque? Dans la onzième élégie, par exemple, Thamire raconte un songe funeste où elle s'imagine assister à la mort de son époux :

À peine je dormois. D'un combat furieux Le spectacle sanglant s'est offert à mes yeux; Cent Tonerres d'airain avec des bruits horribles Vomissent mille morts de leurs bouches terribles;

11 Michault (1734, p. 75-76) qualifie le passage de «désordre furieux». Quelques pages plus loin, à l'inverse, il considère comme élégiaque l'apostrophe à la nuit qui ouvre l'Électre de Crébillon (voir p. 81). 
La fureur \& l'effroi volent de rangs en rangs;

Et je ne vois par tout que morts \& que mourans.

(Le Blanc, 1731, p. 119)

L'hypotypose martiale roule littéralement sur les chevilles héroïco-tragiques : "combat furieux», «spectacle sanglant», «Tonerres d'airain», "Vomissent mille morts», «bouches terribles », " fureur \& [...] effroi », "volent de rangs en rangs », «morts \& [...] mourants». Le registre s'éloigne de la douce mélancolie, voire des saillies somme toute tempérées que l'on rencontre dans le reste du recueil. Et que penser de cette autre héroïne de Le Blanc, qui s'imagine devenir une nouvelle Marianne ou une nouvelle Agrippine :

Oüi, si-tôt que la Parque aura fini mes jours, Cruel! je veux des tiens empoisonner le cours. Ardente à te poursuivre ainsi qu'une Furie, Je te reprocherai ma mort, ta barbarie.

[...]

Mon Ombre à tous momens marchera sur tes pas,

Tu voudras l'éviter : tu ne le pourras pas.

Je serai ton Boureau. (Le Blanc, 1731, p. 75)

Si les imprécations contre les amants infidèles ou insensibles appartiennent effectivement au répertoire des procédés rhétoriques de l'élégie, la tonalité du passage évoque plutôt les planches de la Comédie-Française. En outre, le motif revient dans le sixième poème :

Que je souffre à présent! Furieuse, éperduë, Cruel! de quelque part que je tourne la vûë,

Tout retrace à mes yeux ton crime. Quelle horreur!

Tout... Non, je ne puis plus contenir ma fureur.

Noires Divinitez, qui sur les rives sombres

Tourmentez pour toûjours les criminelles Ombres, Répandez dans mon cœur vos funestes poisons. 
La constellation lexicale qui se rapporte à la fois aux Enfers, à la fureur et à la magie noire rappelle ici davantage la Médée sénéquienne que Délie, Cynthie ou même les héroïdes ovidiennes, incluant la magicienne de Colchos. On objectera que des descriptions infernales se trouvent dans les élégies latines, notamment dans celles de Tibulle. Toutefois, l'abbé Le Blanc ne justifie pas son esthétique par le recours à l'autorité antique; qui plus est, les passages en question ont souvent été critiqués par la tradition critique néolatine et n'ont guère eu de postérité à l'âge classique.

Or, plus que les motifs, c'est la forme même des élégies de Le Blanc qui porte des marques de théâtralité. Certes, la majeure partie d'entre elles mettent en scène de manière plus ou moins explicite un acte d'écriture afin d'assurer la vraisemblance de l'énonciation poétique, en parfait accord avec les règles du genre. Les écarts n'en sont que plus saillants. Ainsi, lors de la finale de la troisième élégie, l'illusion épistolaire est brisée par le morcellement du discours :

Mais que sens-je? Déjà ma force m'abandonne...

Je ne puis... Ah voici mon funeste moment!

J'expire, cher Alcippe, \& j'expire en t'aimant... (Le Blanc, 1731, p. 79)

Le fond répond peut-être à la mise en scène de l'incipit du poème ${ }^{12}$, mais la série d'aposiopèses qui scandent les vers mime ostensiblement les dialogues théâtraux. Un procédé similaire est employé dans la huitième élégie. Cette fois, il ne s'agit plus de mourir sur les planches, mais de quitter la scène :

12 Le Blanc (1731), p. 76 : «Prête à voir de Pluton le ténébreux Empire, / Je n'aurois jamais cru que je pûsse t'écrire, / Cher Amant. Toutes fois je le fais malgré moi. » 
Éloignons-nos plûtôt de ces funestes Lieux : Je suis perdüe hélas! s'il paroît à mes yeux.

Lui pourrois-je... Ah! cherchons mon Salut dans la fuite.

D'un aveu si honteux quelle seroit la suite?

Fuïons... S'il ne venoit peindre encor ses malheurs,

Fuïons... Je ne pourrois résister à ses pleurs. (Le Blanc, 1731, p. 104)

De nouveau, Le Blanc multiplie les aposiopèses et les marqueurs d'oralité : la reprise anaphorique de "Fuïons» accentue l'urgence de la situation et crée une véritable tension dramatique. Les vers finaux sont, quant à eux, explicites : "Je cherche en vain... Qu'entends-je? Ah! C'est lui que je voi! / Je me trouble. Grands Dieux! Je ne suis plus à moi. » (Le Blanc, 1731, p.105) Il ne manque plus que les indications scéniques pour transformer le poème en tirade théâtrale.

Cette esthétique, singulière au plus haut point, atteint son apogée dans la dixième élégie. Après que Phénice s'est adressée à Albine, qu'on suppose être sa confidente, dès le sixième vers, elle lui ordonne de transmettre un message à son amant :

Cher Albine; [...]

Vas le désabuser. Dis-lui combien de larmes...

Hé tu ne vois que trop l'excès de mes allarmes!

Vas, cours, vole, reviens. J'attendrai ton retour. (Le Blanc, 1731, p. 112)

Moins sublime que le «Va, cours, vole et nous venge » du Cid, le passage fonctionne comme une véritable didascalie interne et n'enfreint pas au demeurant les règles classiques de la sortie de scène d'un personnage secondaire. Quelques vers plus loin, l'inquiétude qui gagne l'amante se traduit par un «Que fais Albine? " Cependant, c'est la finale qui opère la dernière rupture. En effet, après un dernier vers plutôt prosaïque, « Je ne devrois plus vivre, \& je ne puis mourir» (Le Blanc, 1731, p.115), Le Blanc ajoute un épilogue en italique à mi-chemin entre la didascalie et le dénouement d'une saynète : 


\section{Albine alors revint, \& la tendre Phénice De ses plaintes enfin reconnut l'injustice. Les Dieux ont tôt ou tard pitié des malheureux. Et c'est toûjours à tort que nous nous plaignons d'eux. (Le Blanc, 1731, p. 116)}

L'élégie s'avère en l'occurrence un monologue en bonne et due forme: le procédé abolit la dernière frontière qui faisait du poème élégiaque un genre lyrique et non dramatique.

Trois ans après la parution de l'ouvrage de Le Blanc, l'abbé Jean-Bernard Michault fait paraître un traité qui contredit presque une par une les propositions de son prédécesseur. Les titres de certains chapitres balisent clairement les enjeux d'un débat sur lequel nous ne pouvons nous attarder ici : «Si l'Élégie peut être Héroïque? CHAP. III», « Si l’Élégie peut être Dramatique? CHAP. IV», "Si les fureurs Tragiques peuvent convenir à l'Élégie? CHAP.V». Soulignons seulement deux particularités de la démarche de Michault. D'une part, il s'agit d'un traité théorique qui ne contient aucune élégie et, d'autre part, l'auteur recourt aux mêmes sophismes afin d'établir sa poétique : il multiplie les comparaisons avec d'autres genres et inclut un nombre considérable de poèmes qui ne sont pas des élégies dans le corpus élégiaque ${ }^{13}$.

13 Voir Delphine Denis (2008), art. cit., p. 70 sq. Une telle méthode se retrouve de surcroît chez Rémond de Saint-Mard, chez Nivernais, dans les articles de l'Encyclopédie rédigés par Marmontel et par Joubert, pour ne nommer que ceux-là. 
N'en déplaise à ses détracteurs - le «Discours sur l'élégie » est aussi vivement critiqué dans Le journal des savants -, Le Blanc anticipe ainsi la mode des héroïdes qui gagne la seconde moitié du XVIIIe siècle ${ }^{14}$ et cause une forte réaction chez des auteurs comme Chénier, Parny, Millevoye, Treneuil, etc. Certes, les poètes et la critique attribueront pendant longtemps l'échec de la véritable élégie - si un tel poème existe - aux formes mêmes de la sensibilité et de la sociabilité sous l'Ancien Régime, plus particulièrement au XVIIIe siècle, comme en témoigne ce propos d'un spécialiste de l'élégie préromantique: «La pensée fait sa toilette. Fontenelle met l'astronomie à la portée des dames. Montesquieu fait de l'esprit sur les lois. » (Henri Potez, 1898, p. 2415) Les impératifs de naturel, de simplicité, de douceur, si fortement liés au style associé à l'élégie, varient d'un siècle à l'autre et, avec eux, l'appréciation des poèmes en question. En revanche, si l'élégie s'avère encore aujourd'hui un genre limitrophe aux frontières poreuses, c'est en grande partie parce que les théoriciens ont rencontré plusieurs difficultés lorsqu'ils ont tenté d'en tracer les limites. "Elle ne doit être distinguée des autres especes de Poësie que par le fond, par l'amour \& par l'amour malheureux » (1731, p. 115), précise Le Blanc, donnant ainsi l'exemple d'une réflexion appelée à verser dans l'imprécision quand on connaît la fortune des tourments amoureux comme sujet de prédilection en littérature.

\footnotetext{
14 Voir Renata Carocci (1988).

15 L'auteur reprend le bon mot attribué à Madame Du Deffand. Le jugement qu'il porte sur des poètes comme Chaulieu et La Fare n'est guère plus tendre : «Cela ne dépasse pas la mélancolie païenne. Après avoir joui de l'amour, des festins et du soleil, ces viveurs attendent sans trop de tristesse la grande nuit » (Potez, 1898, p. 7).
} 


\section{Bibliographie}

Aigaliers, Pierre de Laudun d'. (2000), L'Art poétique françois, éd. J.-C. Monferran, Paris, Société des textes français modernes.

Bolleau, Nicolas. (1998), Art poétique. Épîtres, odes, poésies diverses et épigrammes, éd. S. Menant. Paris, GarnierFlammarion.

CARocCI, Renata. (1988), Les Hérö̈des dans la seconde moitié du XVIII siècle (1758-1788), Paris / Schena, Fasano / A. G. Nizet, coll. « Cultura straniera ».

Chappuis SAndoz, Laure. (2011), Au-delà de l'élégie d'amour. Métamorphoses et renouvellements d'un genre latin dans l'Antiquité et à la Renaissance, Paris, Classiques Garnier.

DENIS, Delphine. (2008), «De l'élégie à l'élégiaque: un débat théorique à l'âge classique », dans Lucille GAUDIN-BORDES et Geneviève SALVAN (dir.), Les Registres. Enjeux stylistiques et visées pragmatiques. Hommage à Anna Jaubert, Louvain LaNeuve, Bruylant / Academia, coll. "Au cœur des textes », p. 65-78.

Dion, Nicholas. (2013), « Muses élégiaques, muses pérennes. La tristesse comme critère définitoire de l'élégie française, de Sébillet (1548) à La Mesnardière (1640)», dans Éric VAN DER SCHUEREN et Matthieu FoRTIN (dir.), De la permanence. Études offertes à Bernard Beugnot pour son quatre-vingtième anniversaire, Paris, Hermann, coll. «République des Lettres / Symposiums », p. 65-84. 
-. (2012), Entre les larmes et l'effroi. La tragédie classique française, 1677-1726, Paris, Classiques Garnier, coll. « Lire le XVIIe siècle ».

FontenElLE, Bernard Le Bovier de. (1969), «Description de l'empire de la poésie », dans Raymond PICARD (dir.), La Poésie française de 1640 à 1680, Paris, S.E.D.E.S., p. 22-24.

-. (1766), "Description de l'empire de la poésie», dans CEuvres, vol. IX, Paris, Libraires associés, p. 438.

FraGuiER, Claude-François. (1729), « Mémoire sur l'élégie grecque et latine », dans Mémoires de littératures tirez des registres de l'Académie royale des inscriptions et belles lettres, vol. VI, Paris, Imprimerie royale, p. 277-282.

GOYET, Francis (dir.). (1990), Traités de poétique et de rhétorique de la Renaissance, Paris, Librairie générale française, coll. «Livre de poche».

LE BLANC, Jean-Bernard. (1731), Élégies [...], avec un discours sur ce genre de poésie, Paris, Chaubert.

MichaULT, Jean-Bernard. (1734), Réflexions critiques sur l'élégie, Dijon, Augé.

PoteZ, Henri. (1898), L'Élégie en France avant le Romantisme, Paris, Calmann-Lévy.

VAUQuelin de LA FReSnaye, Jean. (1885), L’Art poétique, éd. G. Pellissier, Paris, Garnier frères. 


\title{
Résumé
}

Cet article se penche sur le "Discours sur l'élégie», le traité théorique placé en tête des Élégies (1731) de l'abbé JeanBernard Le Blanc, afin de montrer dans quelle mesure il estompe les distinctions génériques alors qu'il devait plutôt assurer leur valeur discriminante. Nos conclusions sont par la suite étendues aux douze élégies qui se veulent un essai de repousser les frontières imposées au genre élégiaque dans la tradition française telle que perçue et analysée par l'abbé Le Blanc et qui partagent de surprenantes affinités avec la poésie dramatique.

\begin{abstract}
This article investigates the "Discours sur l'élégie», the theoretical treatise placed at the beginning of abbé JeanBernard Le Blanc's Élégies (1731), and shows how it blurs generic distinctions instead of ensuring their discriminating role. Those conclusions are subsequently applied to a study of the twelve elegies, which tend to push the boundaries of the elegiac genre set by the French tradition, as abbé Le Blanc understands and analyses it, and which share some surprising similarities with dramatic poetry.
\end{abstract}

